\title{
PD-L1/PD-1 Inhibitors and Beyond: The Evolving Role of Checkpoint Inhibitors and Biomarkers in Cancer Immunotherapy
}

\author{
David J Segarnick ${ }^{1 *}$ and Heather Chien ${ }^{2}$ \\ ${ }^{1}$ Department of Pharmacology, Physiology and Neuroscience, Rutgers New Jersey Medical School/Graduate School of Biomedical Sciences, USA \\ ${ }^{2}$ Research Associate, MedEvoke (a Medisys Health Communications company), USA
}

Submission: December 19, 2016; Published: January 19, 2017

*Corresponding author: David J Segarnick, PhD, Chief Medical Officer, MedEvoke (a Medisys Health Communications company), High Bridge, New Jersey; Department of Pharmacology, Physiology \& Neuroscience, Rutgers New Jersey Medical School/Graduate School of Biomedical Sciences, Newark, New Jersey, USA, Tel: 201-919-1202; Email: segarndj@njms.rutgers.edu

\begin{abstract}
Abbreviations: TME: Tumor Microenvironment; CAR-T: Chimeric Antigen Receptor T cell; CARs: Chimeric Antigen Receptors; ALL: Acute Lymphocytic Leukemia; IHC: Immunohistochemistry; SOSV: Share of Scientific Voice; SSA: Scientific Sentiment Analysis; DCs: Dendritic Cells; CIT: Cancer Immunotherapy
\end{abstract}

\section{Overview}

The past five years have witnessed significant advances in cancer immunotherapeutics, characterized by breakthroughs in response not seen previously with standard chemotherapy. Researchers have studied immunotherapy as potential cancer treatment for decades, but positive large scale clinical trial outcomes have been achieved only recently. When Science magazine declared cancer immunotherapy its 2013 "Breakthrough of the Year," the promise of dramatic therapeutic responses triggered rapid growth in the pace of foundational research, clinical testing, and commercialization of new molecules [1]. Four immunotherapeutic drugs are furthest along in clinical development at this time. Nivolumab and pembrolizumab inhibit PD-1 directly, whereas atezolizumab (MPDL3280A) and MEDI4736 inhibit PD-L1. PD-L1, or programmed death ligand-1, is an inhibitory immune checkpoint molecule found on $\mathrm{T}$ cells that binds the PD-1 receptor to reduce cytokine production and suppress T cell proliferation [2]. Since PD-L1 is often overexpressed in solid tumors, inhibition of the PD-1 pathway may be a viable option to target this mechanism of immune evasion. Tables $1 \& 2$ provide an overview of the clinical activity of these drugs when used as single agents and in combination [3]. These drugs are also being extensively studied in comparison trials versus chemotherapy as well as in combination trials with other systemic and targeted therapies, and other immunotherapies.

Table 1: Majority abstracts/articles covered monotherapy and combination trials*

\begin{tabular}{|c|c|c|c|c|}
\hline & atezolizumab & durvalumab & nivolumab & pembrolizumab \\
\hline Monotherapy & 20 & 17 & 14 & 6 \\
\hline Combination & 2 & 5 & 2 & 1 \\
\hline Total & 22 & 22 & 16 & 7 \\
\hline
\end{tabular}

Table 2: PD-1 and PD-L1 combinations in representative clinical trials*

\begin{tabular}{|c|c|c|c|c|}
\hline & atezolizumab & durvalumab & nivolumab & pembrolizumab \\
\hline Chemotherapy & & & & \\
\hline Anti-CTLA4 & & & & \\
\hline Anti-VEGF & & & & \\
\hline Anti-CD40 & & & & \\
\hline
\end{tabular}




\section{Journal of Pharmacology \& Clinical Research}

\begin{tabular}{|c|l|l|l|l|}
\hline Anti-CD20 & & & & \\
\hline Anti-CCR4 & & & & \\
\hline Anti-OX40 & & & & \\
\hline Anti-HER2 & & & & \\
\hline Anti-ALK & & & & \\
\hline Anti-EGFR & & & & \\
\hline Anti-MEK & & & & \\
\hline Anti-BRAF & & & & \\
\hline Anti-IDO & & & & \\
\hline Anti-KIR & & & & \\
\hline Anti-BTK & & & & \\
\hline Anti-JAK & & & & \\
\hline Anti-LAG3 & & & & \\
\hline AnTI-PARP & & & & \\
\hline
\end{tabular}

${ }^{*}$ Note: Data for Tables $1 \& 2$ obtained and analyzed by Medmeme ${ }^{\mathrm{TM}}$ Impactmeme solution.

Shaded cells in Table 2 indicate the drug combinations featured in the analyzed abstracts.

Some remarkable results have been achieved in highly pre-treated, refractory patients [4]. At the same time, PD-1/ PD-L1 inhibition, successful as it has been, reflects just the tip of the iceberg among many new immunotherapeutic agents with different mechanisms of action in the pipeline that will significantly alter the oncology therapeutic landscape over the next five years. While the aforementioned agents are furthest along in development, they are merely an indication of what is yet to come. In the following pages we will share our observations from a cancer immunotherapy congress we attended in Massachusetts and highlight issues and important trends [5]. We will provide an overlay to these observations and trends using our proprietary share of scientific voice and sentiment analysis methodologies and analytical tools. Finally, to provide greater insight into future directions in cancer immunotherapy we will discuss the alignment between the trends we observed at the Massachusetts congress and our analysis of the published literature and other congress abstracts.

\section{A personalized approach to combination immunotherapy}

According to Carol O'Hear, MD, PhD, Assistant Director, Cancer Immunotherapy at Genentech who presented her findings on "Personalized Approach to Combination Immunotherapy" at the Cancer Immunotherapy Congress [5] in Wakefield, Massachusetts on May 19 - 20, 2016, PD-L1/PD-1 antitumor effects work best in "inflamed tumors" as opposed to noninflamed tumors. Inflamed tumors are characterized by high PDL1 levels, cellular inflammation, and pre-existing immunity. The greatest responses to PD-L1 agents, such as atezolizumab, have been in combination with chemotherapeutic agents that work synergistically to inflame the tumor, thereby facilitating PD-L1 effects via migration of CD8+ T cells into the tumor cells. The theory is that concomitant (or potentially immediately prior) exposure to standard chemotherapy primes the tumor to allow $\mathrm{T}$ cells to better penetrate into the tumor microenvironment (TME). Further, a high mutational tumor load is a predictor of response to checkpoint inhibitors (independent of PD-L1 expression).

Another hot topic at the Cancer Immunotherapy Congress was "Efficacy and Safety of Combined MAPK Targeted Therapy and Immunotherapy in Patients With Melanoma" by Ryan Sullivan, MD, Harvard Medical School, who described the emerging importance of using dual targeting in melanoma with anti-PD-L1 and RAS inhibitors [5]. Studies are underway looking at a lead-in period with a RAS/BRAF inhibitor, followed by combination RAS/BRAF + anti-PD-L1 (e.g., pembrolizumab) for a month, followed by longer term follow-up with mono-PD-L1 therapy. The theory is that the RAS inhibitor primes the tumor by increasing antigen expression, decreases immunosuppression, increases the number of CD8+ $\mathrm{T}$ cells and improves $\mathrm{T}$ cell infiltration and clonality.

\section{Chimeric antigen receptor $T$ cell technology (CAR-T cell immunotherapy)}

Several companies are in phase 3 development of CAR-T (chimeric antigen receptor $\mathrm{T}$ cell) technology, and these data were discussed throughout the conference [5]. Artificial T cell receptors (also known as chimeric $\mathrm{T}$ cell receptors, chimeric immunoreceptors, or chimeric antigen receptors (CARs) are engineered receptors that allow the $\mathrm{T}$ cells to recognize a specific protein antigen on a tumor cell and attack these cancer cells. The receptors are called chimeric because they are composed of parts from different sources. Typically, these receptors are used to bind the monoclonal antibody onto a $\mathrm{T}$ cell, with transfer of their coding sequence facilitated by retroviral vectors.

The process is a true pharma/hospital linked therapeutic agent/process whereby the patient's blood is drawn, separated, treated, activated and then reinfused to allow the CAR-T cells to attack the tumor, essentially allowing the body's own (modified) immune cells to attack the foreign antigen. Response rates achieved in acute lymphocytic leukemia (ALL) are greater than 
that seen with current standard of care therapy, greater than a 90 percent overall response rate [6]. Figure 1 illustrates the interest of the scientific community surrounding CAR-T therapy.

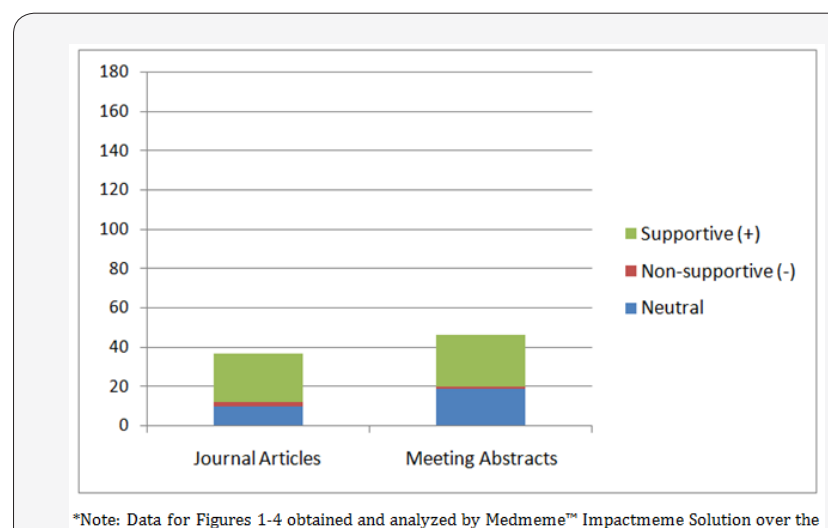
last full year (2015).

Figure 1: CAR-T therapy sentiment by source.

\section{Biomarkers in cancer immunotherapy}

Another key theme that emerged from the congress concerned the role of biomarkers in patient selection and cancer cell targeting. Two presentations focused attention on the biomarker concept: "Biomarkers and Immune Correlates of Clinical Outcome" delivered by James Trager, PhD, VP Research, Dendreon Pharma as well as "Tumor Immunology and Oncology: Biomarkers and Personalized Immunotherapy" by Dr. Jianda Yuan, Director, Translational Research, Merck [5]. The detection of appropriate biomarkers for each tumor type drives T cells into tumors and produces increased antigen uptake, APC (antigen presenting cell) activation, antibody humoral response and antigen spread. Taken together, these investigators are exploring ways to improve response rates beyond PD-L1 expression, including the development of novel whole exon sequencing, whole genome sequencing, immunohistochemistry (IHC), B and $\mathrm{T}$ cell function.

\section{Dendritic cell therapy}

As the most prominent antigen-presenting cells and the sentinels of the immune system, dendritic cells (DCs) are of significant interest among the scientific community. According to Dr. Joshua Brody, Director, Lymphoma Immunotherapy Program, Mount Sinai School of Medicine, dendritic cell-based vaccination is currently being investigated as a promising approach [5]. Results from many clinical trials have shown that dendritic cell vaccination can reverse the immune suppressed tumor micro-environment as well as activate antitumor immune responses. DC-vaccination involves extracting dendritic cells from the patient's blood and exposing them to tumor antigen. The subsequent antigen processing allows for the maturation of the dendritic cells, which can then be injected back into the patient to activate T lymphocytes. Promising results (Figure 2) have driven an increase in studies of this treatment modality.
Figure 3 shows that DC therapy is currently the second most frequently discussed emerging cancer immunotherapy strategy.

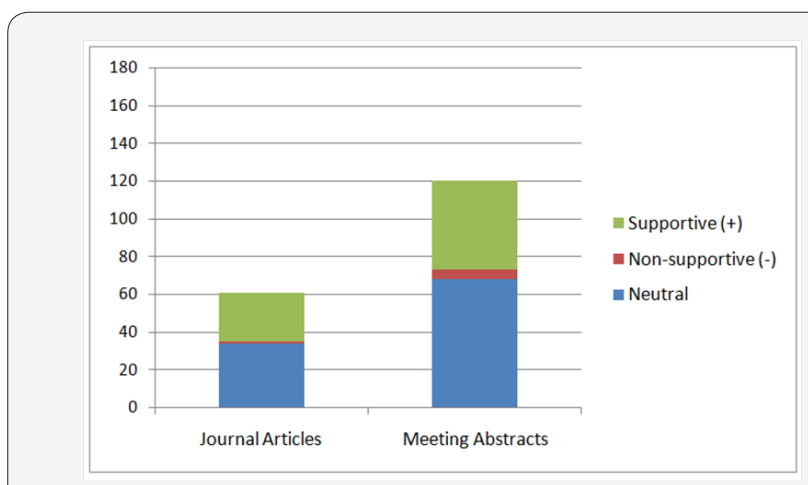

Figure 2: DC therapy sentiment by source.

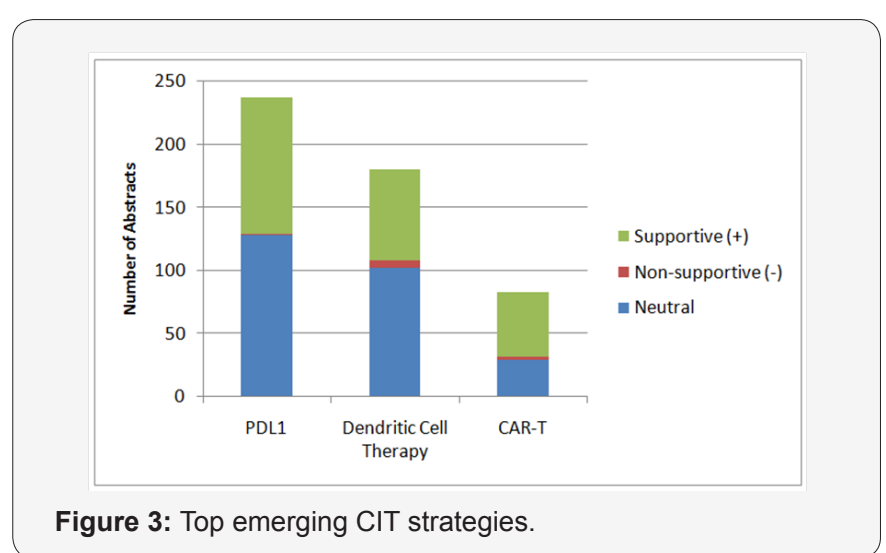

\section{The future of cancer immunotherapy (CIT): Current sentiment and future direction}

To further analyze these data, we applied analytical tools and proprietary share of scientific voice (SOSV) and scientific sentiment analysis (SSA) methodologies developed by MedEvoke (High Bridge, NJ) to evaluate the scientific community's interest and adoption of the aforementioned therapies via a broad search of the Medmeme ${ }^{\mathrm{TM}}$ information and analytical tools platforms (e.g., Insightmeme and Impactmeme) [3]. Figure 3 shows the volume of peer-reviewed data (publications and meeting abstracts) pertinent to the three most highly investigated emerging cancer immunotherapies over the last full year (2015). To add qualitative value, MedEvoke evaluated and categorized each abstract according to the author's sentiment towards the specified treatment modalities. Not surprisingly, PD-L1 has been the immunotherapy option discussed most often at congress meetings and in journal publications. Dendritic and CAR-T therapies have also been generating interest with increasing uptake.

Of all relevant abstracts, the sentiment surrounding these top emerging cancer immunotherapy strategies is overwhelmingly supportive. Most abstracts or articles displaying non-supportive sentiment can be attributed to concern over safety (e.g., cytokine 
storm) or efficacy issues as well as improvements that can be made to these therapies. As is typical with novel therapies, further research is necessary to elucidate possible adverse effects and to refine these promising treatment modalities. We also grouped the volume associated with each strategy by source to reveal additional insights. Figure 4 shows that despite having been in the scientific discourse for the longest period of time, PD-L1 therapy continues to be the subject of the greatest volume of congress activity, suggesting that investigator interest has not yet peaked. While Figure 2 shows that the volume of meeting abstracts mentioning DC therapy is substantially less than for PD-L1, the volume of journal mentions is nearly equal, indicating faster conversion from congress "news" to publication. Figure 1 indicates that CAR-T therapy is still a relatively new concept, but that findings revealed at congresses are appearing in journals quickly thereafter.

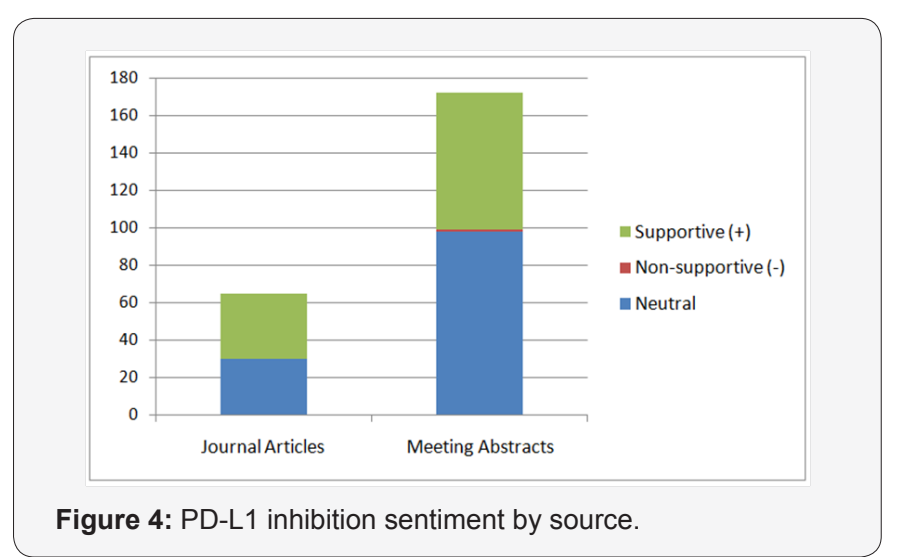

\section{Conclusion}

The question asked most frequently at the conference was: "Where is the field heading beyond anti-PD-L1 agents?"
While results have been remarkable with these agents to date, particularly in combination therapy, the presenters agreed that there are certainly other checkpoint inhibitors that may "remove the brakes" from the immune system in even more effective ways. Some of the new cancer immunotherapy targets include: cytokines (IL-2, next generation interferons), therapeutic cancer vaccines, oncolytic viruses, adjuvants (RNA surrogates), cell therapies (e.g., CAR-T), TNK (CD-19+ targeted), TAMs and Tregs, APC activators (e.g., dendritic cell therapy) and other checkpoint modulators (beyond PD-L1/PD-1) [5]. The expectation is that these emerging cancer immunotherapy approaches will increase tumor priming; stimulate $\mathrm{T}$ cell activity and tumor killing, and further impact $\mathrm{T}$ cell modification. Newly gained insights are allowing for renewed enthusiasm in industry as well as in academia. No doubt that, while the field is only five years old, it has already produced unprecedented clinical results that mark just the beginning of this revolution with dendritic cell therapy and CAR-T next on the therapeutic horizon.

\section{References}

1. Couzin-Frankel J (2013) Breakthrough of the year 2013. Cancer immunotherapy. Science 342(6165): 1432-1433.

2. Topalian SL, Drake CG, Pardoll DM (2012) Targeting the PD-1/B7-H1 (PD-L1) pathway to activate antitumor immunity. Curr Opin Immunol 24(2): 207-212.

3. (2015) Data for all tables from Medmeme ${ }^{\mathrm{TM}}$ Impactmeme Solutions, New York, USA.

4. Anshu Giri, Simrit S Walia, Ajeet Gajra (2016) Clinical Trials Investigating Immune Checkpoint Inhibitors in Non-Small-Cell Lung Cancer. Rev Recent Clin Trials 11(4): 297-305.

5. (2016) Cancer immunotherapy congress. Boston, USA.

6. Maus MV, June CH (2016) Making Better Chimeric Antigen Receptors for Adoptive T cell Therapy. Clin Cancer Res 22(8): 1875-1884.

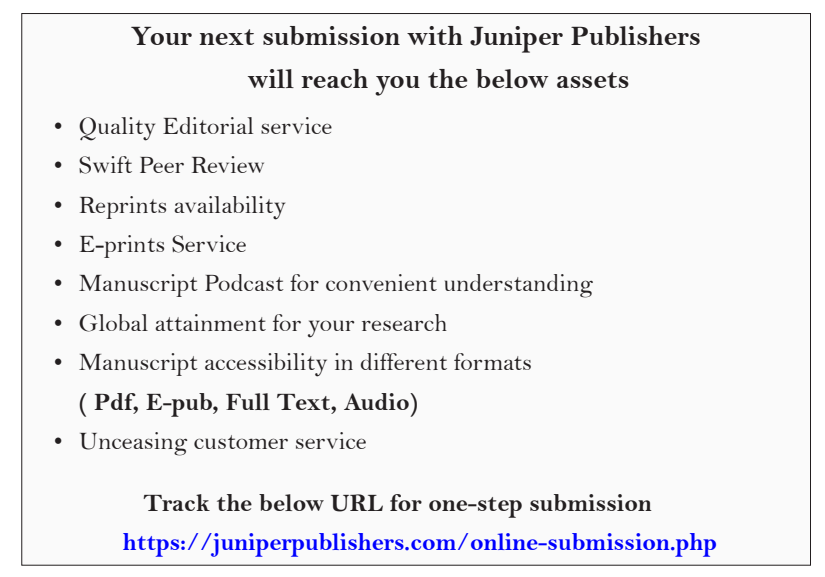

